\title{
El proyecto de investigación del desarrollo didáctico de la expresión y el arte (IDDEA): pedagogía del arte en educación superior
}

Sonia Rodríguez-Cano, Vanesa Delgado-Benito y Vanesa Ausín-Villaverde

Universidad de Burgos, Facultad de Educación, Departamento Ciencias de la Educación, Calle Villadiego, 1. 09001.

Burgos-España (correo-e: srcano@ubu.es; vdelgado@ubu.es; vausin@ubu.es)

Recibido Abr. 7, 2020; Aceptado Jun.9, 2020; Versión final Jul. 22, 2020, Publicado Dic. 2020

\begin{abstract}
Resumen
Este estudio presenta una experiencia de innovación docente utilizando la pedagogía del arte enmarcada en el proyecto IDDEA (investigación del desarrollo didáctico de la expresión y el arte). Los estudiantes participantes $(n=26)$, pertenecientes al grado en pedagogía, diseñaron y produjeron un material didáctico audiovisual para trabajar la coeducación y el fomento de valores de respeto e igualdad. Se inspiraron en la obra artística de la pintora Ángeles Romo. Un total de siete píldoras formativas audiovisuales fueron creadas con la técnica de animación Cut-Out. Los resultados muestran que los futuros pedagogos se sienten satisfechos con la utilización del arte como recurso didáctico y manifiestan que utilizarán esta estrategia en su futuro profesional. Las docentes implicadas consideran el gran potencial pedagógico del arte en el ámbito educativo, concretamente, la implementación de la pedagogía del arte en el contexto de educación superior. Se concluye que esta ha contribuido a conseguir los objetivos curriculares en la experiencia pedagógica realizada.
\end{abstract}

Palabras clave: pedagogía; arte; Cut-Out; píldoras formativas audiovisuales; educación superior

\section{The investigation of the didactic development of expression and art (IDDEA) project: art pedagogy in higher education.}

\begin{abstract}
This article presents an experience of teaching innovation using art pedagogy framed in the IDDEA (investigation of the didactic development of expression and art) project. The participating students $(n=26)$ attending a pedagogy degree designed and produced an audiovisual teaching material to work on coeducation and the promotion of values (respect and equality). The students were inspired by the artistic work of the painter Ángeles Romo. Seven audiovisual training pills were created by using the Cut-Out animation technique. The results show that the students (future educators) felt satisfied with the use of art as a teaching resource and stated that they would use this strategy in their professional careers. The professors involved considered the great pedagogical potential of art in the educational field, specially, the implementation of art pedagogy in the context of higher education. It is concluded that art pedagogy has contributed to achieving the curricular objectives in the pedagogy experience presented here.
\end{abstract}




\section{INTRODUCCIÓN}

En 2006 el pedagogo británico Ken Robinson, con su charla TED "¿las escuelas matan la creatividad?", planteaba de manera entretenida y conmovedora la necesidad de crear un sistema educativo que nutra la creatividad en vez de matarla (Robinson y Aronica, 2019). Hablar sobre los aprendizajes y las enseñanzas que tienen en cuenta el potencial creativo, entendiendo como tal el lenguaje artístico y la expresión visual, es un tema recurrente en las investigaciones sobre educación de los últimos tiempos (Holdhus, 2019; Jeffrey y Craft, 2004; París, 2020; Pavlou, 2020; Suárez et al., 2019). Al respecto, un estudio desarrollado por Vidagañ et al. (2016) hace patente que se pueden trabajar otros conceptos educativos a través del arte, como la educación expandida, los aprendizajes colaterales así como el currículum oculto. Autores como Berrocal et al. (2010) defienden que los profesionales de la educación debieran desplegar un mayor esfuerzo en su quehacer profesional para contribuir al aprendizaje significativo de los estudiantes, a la vez que desarrollan el potencial creativo de estos mediante la utilización de los distintos lenguajes artísticos.

Teniendo en cuenta el escenario educativo en el que nos encontramos, cuando hablamos de la formación inicial de los futuros pedagogos de este siglo, coincidimos con la idea de Mora y Osses (2012), quienes proponen que el arte, desde una perspectiva pedagógica, se convierte en una poderosa herramienta didáctica que facilita el desarrollo subjetivo del conocimiento y las potencialidades humanas. En este sentido, la educación superior es un excelente escenario para utilizar la creatividad (Hennessey, 2010) y así, contribuir a que los profesionales del futuro desarrollen conocimientos y habilidades de investigación, cooperación, conexión e integración de los saberes con las competencias profesionales (Livingston, 2010).

Experiencias como la desarrollada por Menéndez-Varela y Grigori-Giralt (2017) a partir de la metodología aprendizaje servicio para la construcción de la identidad profesional de los estudiantes universitarios a través del arte es un ejemplo ya que sus resultados han demostrado cómo es posible que los estudiantes construyan una identidad personal y social basada en el compromiso emocional hacia su profesión y con la sociedad. Como se puede comprobar la integración de la metodología aprendizaje servicio, responde a los planteamientos que Dewey propuso para trabajar la proyección social de la educación a través de la conexión de las actividades educativas con una experiencia real, cooperativa.

Desde esta perspectiva holística, en el ámbito universitario, una manera de trabajar a través del arte es mediante el diseño y creación de recursos didácticos a partir de la expresión artística (Mundet et al., 2015), pudiendo integrar también la tecnología para su desarrollo (Loveless, 2003; Mayo, 2007). Esta es una vía eficaz de expresión de sentimientos, emociones, sensaciones y vivencias a la vez que supone una oportunidad educativa para el desarrollo personal y social de los estudiantes.

La educación es un universo complejo y la acción de educar no supone trasmitir conocimientos, sino que los estudiantes sean capaces de vivenciar e interiorizar tanto ideas como actitudes y valores. Entendemos la educación desde una perspectiva expandida, que consiste en integrar otros espacios de aprendizaje para nutrir los contenidos de la educación formal, con saberes, reflexiones y perspectivas no formales, que se añaden a los currículums formativos como estrategias de aprendizaje. Sobre esta línea, el propósito de este artículo es mostrar cómo se ha trabajado transversalmente, utilizando la pedagogía del arte, el tema de la igualdad de género a través de la obra pictórica de la artista Ángeles Romo con el alumnado matriculado en el Grado de Pedagogía de la Universidad de Burgos (España). En ese sentido, otras experiencias educativas previas como las desarrolladas por Moreno et al. (2016), en las que se trabaja el tema de la igualdad de género a partir del arte, concluyen que la experiencia ha contribuido al desarrollo de competencias de indagación y cooperación por parte de los estudiantes, a la vez que ha contribuido al aprendizaje informal por parte de los alumnos.

\section{DESARROLLO DEL TEMA}

El desarrollo del tema se presenta en cinco subsecciones: explicación del concepto de pedagogía del arte, descripción del proyecto IDDEA, contextualización de la experiencia educativa realizada, desarrollo de la actividad y, por último, diseño y producción del material audiovisual didáctico.

\section{Pedagogía del arte}

Si la pedagogía es aquella disciplina científica que tiene como fin el estudio de la educación -en sus múltiples dimensiones-, la pedagogía del arte tiene nuevos retos. De acuerdo con Touriñán (2016), el objetivo de la pedagogía es transformar la información en conocimiento y el conocimiento en educación, constituyendo las artes un área de experiencia cultural cognoscible, enseñable, investigable y realizable que puede constituirse en objeto y meta de la educación. 
La pedagogía del arte pretende utilizar las artes para desarrollarlas como ámbito general de educación, como ámbito de educación general y como ámbito de educación vocacional y profesional (Touriñán, 2016: 72-73): 1) Utilizar las artes para generar en los educandos valores vinculados al carácter y al sentido inherentes al significado de educación; 2) Utilizar las artes para generar en los educandos destrezas, hábitos, actitudes, conocimientos y competencias que los capacitan para decidir y realizar su proyecto de vida personal y construirse a sí mismos; 3) Utilizar las artes para desarrollar nuestro sentido estético y artístico y nuestra capacidad de ser espectadores críticos e incipientes realizadores de actividades y objetos artísticos; 4) Utilizar las artes como dominio teórico tecnológico y práctico, que es cognoscible, enseñable, investigable y realizable-practicable, en el que se puede lograr competencia para crear objetos artísticos, utilizando las formas de expresión y los instrumentos apropiados, con sentido vocacional y, si esa fuera nuestra meta laboral, con sentido profesional.

El arte constituye un instrumento de trabajo pedagógico versátil y reflexivo que ayuda al alumno a proyectar ideas y también a recibirlas y recrearse en las de otros (Orr y Shreeve, 2017). El arte, y en concreto la pintura, constituye un diálogo visual en el que se establecen interrelaciones intencionales y no intencionales que enriquecen el proceso didáctico y facilitan los aprendizajes. Los aprendizajes a través de las artes visuales en la escuela se convierten en un proceso transformador que implica la persona en la construcción sociocultural y educativa de conocimientos que amplía al máximo "el desarrollo de capacidades, competencias y generación de actitudes y valores socialmente significativos, para un contexto determinado, en el que los actores del mismo [...], se transforman y transforman la realidad" (Maeso-Rubio, 2008, p. 146). En el contexto escolar, el desarrollo de los profesores, el entorno y el currículo desempeñan un papel fundamental en la potencialización de la creatividad y el talento (Ucus y Acar, 2019). No obstante, recientes investigaciones indican la no comprensión por parte de los docentes de la importancia del desarrollo creatividad, además del desarrollo de creencias sesgadas en torno a la creatividad en el ámbito educativo. Además también se pone de relieve la necesidad de una adecuada formación en creatividad dentro de la capacitación de los profesionales de la educación (Cropley, et al., 2019).

Otro estudio realizado por Winner et al. (2014) sobre la influencia de las habilidades artísticas en los procesos de enseñanza aprendizaje demuestra que hay evidencias en cuanto a la influencia del aprendizaje artístico en la creatividad y en el pensamiento reflexivo, así como en las habilidades sociales y de comportamiento. En este sentido, para poder conseguir la pedagogía artística de forma plena, debemos asumir sus riesgos, desafiar los estándares preestablecidos y cuestionar los paradigmas tradicionales. Es decir, para poder construir en el arte y la pedagogía, hay que estar dispuesto a destruir. A partir de este cuestionamiento, las prácticas educativas comenzarán a tener un sentido propio, permitiendo a los estudiantes establecer una vinculación con el contexto interpersonal y social. Por otro lado, Moreno-González (2010) conceptualiza la mediación artística en relación a los procesos socioeducativos, en los que, a través del arte, la pintura, las artes escénicas, la música, etc. los individuos son capaces de encontrar sus metas y plantearse objetivos personales y sociales.

También cabe destacar un nuevo método al que han denominado Art thinking (Acaso y Megías, 2017) desarrollado por las docentes universitarias María Acaso y Clara Megías, fundamentado en los descubrimientos de la neuroeducación, inspirado en los procesos de creación de arte y ligado a la metodología del pensamiento artístico (Whitaker, 2016). La base científica en la que se asienta el Art Thinking es la neuroeducación, que estudia cómo aprende el cerebro, y cuyo principal hallazgo es que para que se produzca el aprendizaje es necesario encender una emoción y/o despertar la curiosidad del estudiante.

\section{Proyecto IDDEA}

El proyecto IDDEA (Investigación del Desarrollo Didáctico de la Expresión y el Arte) es una iniciativa educativa que nace desde Asire que es una asociación española de profesionales de la educación y padres que realiza proyectos de innovación educativa e investigaciones para el fomento de un desarrollo global. Asire apuesta por una educación integral potenciando, entre otros aspectos, el desarrollo intelectual, personal y social tanto de los alumnos como de los profesionales de la educación. Cabe mencionar que este proyecto se encuentra bajo registro de la propiedad intelectual con referencia BU-30-17.

Tomando como referencia la pedagogía como instrumento para el desarrollo didáctico, IDDEA surge de la necesidad de atender temas sociales y planteamientos escolares desde una perspectiva artística a través de la cual la obra de arte y el artista se convierten en foco didáctico mediante el que se trabajan los diferentes contenidos temáticos. En colaboración con la empresa de automoción Ural motor situada en Burgos (España), la Asociación Asire (2017) propone la obra de la joven artista Ángeles Romo (Sitio web de la artista: http://www.angelesromo.com/) para dotar de contenido a un proyecto educativo sobre igualdad de género.

El proyecto educativo se plantea con un doble objetivo, por un lado, permitir la reflexión sobre la obra de la citada pintora y el desarrollo y la formación de opiniones críticas sobre la situación actual de la mujer en los 
medios de comunicación y la cultura de la imagen tanto en medios escritos como audiovisuales, considerando esta una meta lo suficientemente importante como para fundamentar el proyecto. Por otro lado, planteamos un segundo objetivo más ambicioso, el cual consiste en el diseño y producción de un material didáctico audiovisual que pueda ser utilizado en aulas de Educación Primaria para trabajar la coeducación y el fomento de valores de respeto e igualdad partiendo de la obra de Romo.

Paralelamente al proyecto educativo IDDEA se promovió un concurso de premios como reconocimiento al trabajo realizado por los estudiantes universitarios participantes en esta experiencia educativa. En resumen, las bases establecidas en el concurso fueron las siguientes: 1) el fin didáctico del material hace que se requiera formación pedagógica previa, por lo que podrán presentarse al concurso mayores de edad en formación o con formación didáctica y pedagógica; 2) la participación implica la originalidad y la titularidad del material; 3) posibilidad de participación individual o grupal; 4) el material puede tener cualquier tipo de soporte, tanto digital como soportes más tradicionales; 5) el recurso didáctico creado debe hacer referencia al tema seleccionado por la artista en su exposición; 6 ) en ningún caso se admitirán escenas o textos que vulneren los derechos fundamentales o que se consideren sexistas, violentos o poco adecuados para su utilización en el ámbito escolar. Se concedieron dos premios, un primero de $150 €$ y un segundo de $100 €$. Ambos galardones también conllevan otro reconocimiento asociado que es la publicación del recurso didáctico con registro ISBN. Para la resolución del concurso se constituyó un jurado de expertos compuesto por representantes de Asire, Audi y profesionales de la Educación y el arte.

\section{Contextualización de la experiencia}

La experiencia educativa aquí presentada se ha llevado a cabo en la Facultad de Educación de la Universidad de Burgos, concretamente en la asignatura "Televisión, Medios Audiovisuales y Educación", impartida en tercer curso de Grado en Pedagogía, dentro de la mención cualificadora de Tecnología y Educación. Del total de estudiantes participantes $(n=26)$, la gran mayoría son mujeres $(92,3 \%)$ y su edad media es de 21,53 . Cabe destacar que algunos están cursando un segundo Grado, habiendo realizado un Grado en Magisterio previamente. Los objetivos docentes planteados en la asignatura son diversos, destacando el que hace referencia a "conocer y diseñar recursos socioeducativos adaptados a las nuevas tecnologías (TIC) y a los distintos niveles de aprendizaje tanto en modalidad presencial como virtual", ya que está directamente relacionado con el proyecto educativo IDDEA aquí presentado.

En cuanto a la extensión temporal de la experiencia, fue llevada a cabo durante los meses de octubre y noviembre, coincidiendo con la exposición de la artista en nuestra ciudad.

\section{La participación en el proyecto IDDEA}

En un primer momento se realizó una sesión explicativa con el grupo clase acerca del propósito perseguido en el proyecto IDDEA, durante la sesión estuvo presente la presidenta de la Asociación Asire quien detalló los objetivos del proyecto e invitó al alumnado a participar en el mismo. Tras la explicación, en otra sesión, se llevó a cabo la presentación de la obra artística por parte de su autora, la pintora Ángeles Romo quien invitó a los estudiantes a visitar la exposición, la cual consta de veintitrés obras agrupadas en función de la técnica utilizada: pintura acrílica (Erótica) e imágenes modificadas digitalmente (¿Está en ti?). Llegados a este punto, se pidió a los estudiantes que crearan grupos de trabajo, así como que acudiesen a ver la exposición de la artista. Durante la exposición debían observar las diferentes obras y seleccionar al menos dos que resultasen motivadoras para diseñar y producir el material audiovisual didáctico en relación con la temática de la coeducación y el fomento de valores de respeto e igualdad.

Siguiendo con el desarrollo de la actividad, cada uno de los grupos de trabajo expuso su selección de obras, habiendo algunas coincidencias entre grupos. Finalmente se llegó a un consenso de tal forma que las obras no se repitieran. A modo de orientación, se facilitó a los estudiantes una reflexión sobre la exposición facilitada por la autora. En la Figura 1 se muestran las obras artísticas utilizadas y su denominación.

\section{Diseño y producción del material audiovisual didáctico}

Después de la selección de las obras artísticas por parte de los estudiantes se pidió a los grupos de trabajo que diseñaran y crearan una píldora formativa audiovisual. El objetivo del material diseñado era trabajar la temática de la coeducación y el fomento de valores de respeto e igualdad, todo ello inspirándose en la obra pictórica seleccionada. Por otro lado, la finalidad de estos materiales era su utilización con alumnos escolarizados en Educación Primaria de centros de Burgos para trabajar la sensibilización y la igualdad hacia el tema de género. Tras realizar la reflexión oportuna sobre este tema tan relevante y de obligada presencia en las aulas, diseñaron sus materiales audiovisuales didácticos basándose en la técnica de animación Cut out. 


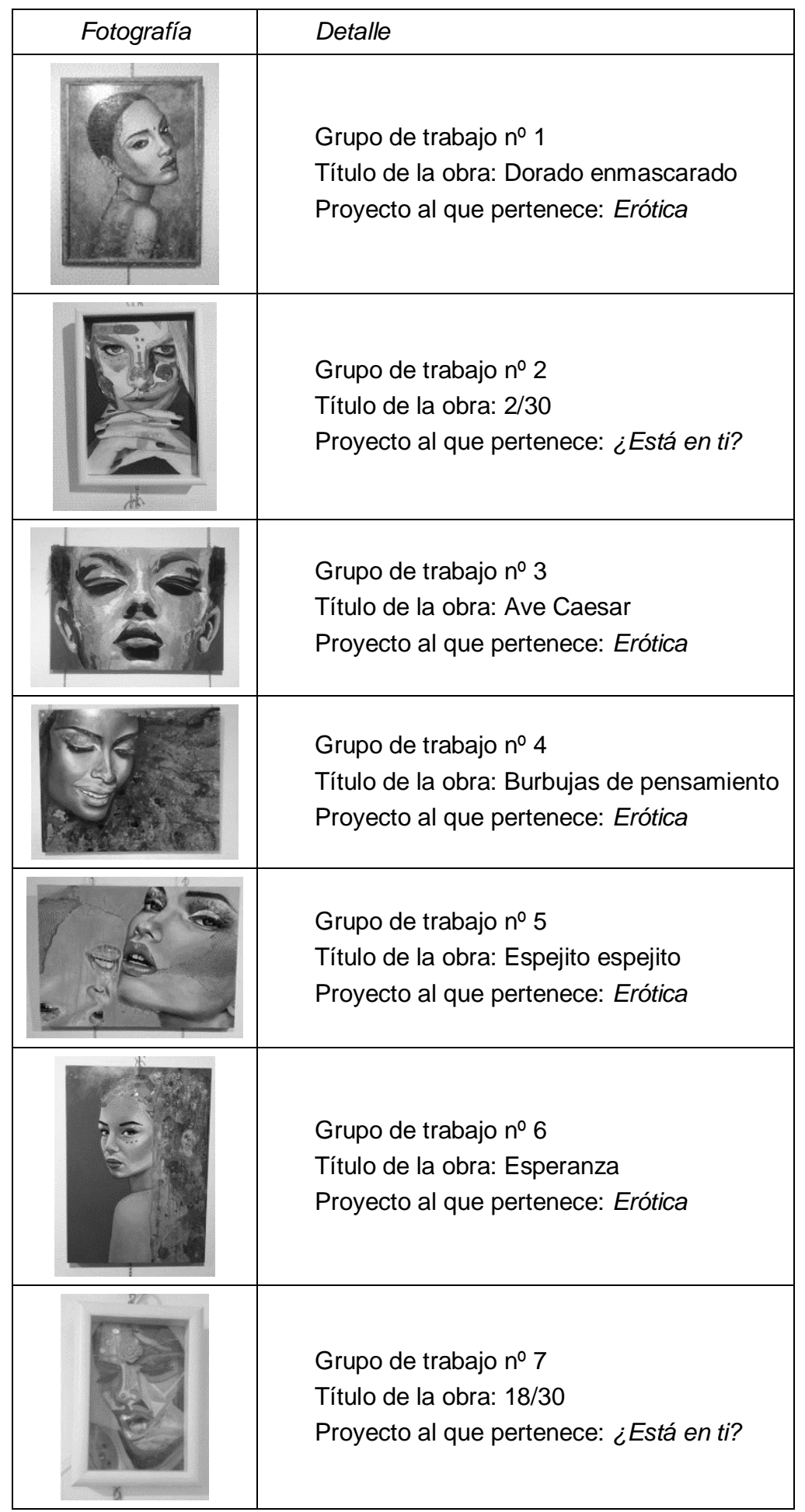

Fig. 1: Obras artísticas seleccionadas para la creación del material audiovisual didáctico (imágenes extraídas, con autorización, de la exposición pictórica de Romo).

La técnica de animación Cut-out es una variante del Stop Motion, que se realiza mediante la utilización de recortes de papel, cartulina, tela o cualquier otro material plano recortado y situado sobre un fondo en el que se moverán los diversos elementos. La sensación de movimiento se consigue mediante la realización de fotografías desde un plano cenital de cada una de las escenografías, es decir, fotograma a fotograma (Delgado-Benito et al., 2015). Así mismo, se les pidió que el material audiovisual creado cumpliera las siguientes características: 1) corta duración: entre 2 y 5 minutos; 2) inserción de audio (música sin letra o voz en off); 3 ) inclusión de créditos (nombres y apellidos de los miembros del grupo de trabajo). Para la edición del vídeo se sugirió la utilización del programa Windows Movie Maker por su sencillez y facilidad de uso, no obstante, se dio a los alumnos total libertad para utilizar el programa de edición que considerasen oportuno. 


\section{RESULTADOS Y DISCUSIÓN}

El fallo del concurso determinó como ganador del primer premio al grupo de trabajo no 1 quienes crearon el material didáctico a partir de la obra "Dorado enmascarado", por otra parte, el segundo premio recayó en el grupo de trabajo $\mathrm{n}^{\circ} 4$ con su trabajo basado en la obra "Burbujas de pensamiento". El objetivo de estas producciones audiovisuales creadas por el alumnado es que se conviertan en un material didáctico que permita vincular el trabajo de los estudiantes de la Facultad de Educación con el trabajo realizado por alumnado de Educación Primaria de diferentes centros educativos. Al finalizar la experiencia, se administró a los estudiantes participantes un breve cuestionario con la finalidad de realizar una valoración sobre la realización de la misma, así como sobre la percepción de futuros profesionales de la educación acerca de la importancia del arte como recurso didáctico. A continuación, resumimos los principales resultados extraídos. Del total de estudiantes participantes $(n=26)$, contestaron al cuestionario el $74,07 \%$, siendo en su totalidad mujeres, con una edad media de 21,7 años.

En un primer momento, se preguntó al alumnado acerca de la técnica de animación Cut out utilizada para la realización del material audiovisual. Al respecto, destacar que las tres cuartas partes conocían previamente en qué consistía la técnica de animación Cut-Out. El $85 \%$ valora como alto o muy alto el grado de interés y motivación previa ante la realización de la actividad. Respecto al resultado obtenido tras su realización, como se puede ver en la Tabla 1, tan sólo en uno de los casos manifiestan estar insatisfechos.

Tabla 1: Grado de satisfacción con el resultado obtenido utilizando la técnica de animación Cut-out

\begin{tabular}{|l|l|c|c|c|c|}
\hline \multicolumn{2}{|c|}{} & Frecuencia & Porcentaje & Porcentaje válido & Porcentaje acumulado \\
\hline \multirow{4}{*}{ Válidos } & Muy satisfecho/a & 6 & 30,0 & 30,0 & 30,0 \\
\cline { 2 - 6 } & Satisfecho/a & 13 & 65,0 & 65,0 & 95,0 \\
\cline { 2 - 6 } & Insatisfecho/a & 1 & 5,0 & 5,0 & 100,0 \\
\cline { 2 - 6 } & Total & 20 & 100,0 & 100,0 & \\
\hline
\end{tabular}

Destacar que, en su totalidad, consideran que esta técnica de animación puede ser útil para su futuro académico y profesional ya que posibilita comprender y asimilar información, enlazar ideas, crear propios contenidos, organizarlos y plasmarlos en formato vídeo. Por tanto, afirman que utilizarán la técnica de animación Cut-Out en un futuro como pedagogos.

Por otro lado, la mitad de los estudiantes (55\%) han utilizado previamente recursos didácticos artísticos en algún proyecto educativo y/o actividad académica. El 95\% de quienes han cumplimentado el cuestionario manifiestan que el arte, en este caso la pintura, ha sido un vehículo adecuado para trabajar pedagógicamente la temática de la igualdad de género en alumnos de Educación Primaria. En igual porcentaje consideran que la motivación por el aprendizaje aumenta cuando utilizamos el arte. Al preguntarles en qué medida consideran que puede resultar motivadora la enseñanza a través del arte, los datos recogidos en la Tabla 2 muestran que ocho de cada diez indican Mucho o Bastante. Centrándonos en la actividad realizada, preguntamos acerca de la contribución de la exposición de pintura de Ángeles Romo en relación a los objetivos planteados en el proyecto. Al respecto encontramos diversidad de opiniones siendo estas polarizadas como puede observarse en la Tabla 3.

Tabla 2: Grado en que consideran que puede ser motivadora la enseñanza a través del arte

\begin{tabular}{|l|l|l|l|l|l|}
\hline \multicolumn{2}{|c|}{} & Frecuencia & Porcentaje & $\begin{array}{l}\text { Porcentaje } \\
\text { válido }\end{array}$ & $\begin{array}{l}\text { Porcentaje } \\
\text { acumulado }\end{array}$ \\
\hline \multirow{4}{*}{ Válidos } & Ni mucho ni poco & 4 & 20,0 & 20,0 & 20,0 \\
\cline { 2 - 6 } & Mucho & 13 & 65,0 & 65,0 & 85,0 \\
\cline { 2 - 6 } & Bastante & 3 & 15,0 & 15,0 & 100,0 \\
\cline { 2 - 6 } & Total & 20 & 100,0 & 100,0 & \\
\hline
\end{tabular}

Tabla 3: Grado en el que la exposición de pintura de Ángeles Romo ha ayudado en la consecución de los objetivos planteados en el proyecto

\begin{tabular}{|l|l|l|l|l|l|}
\hline \multicolumn{2}{|c|}{} & Frecuencia & Porcentaje & Porcentaje válido & Porcentaje acumulado \\
\hline \multirow{4}{*}{ Válidos } & Nada & 2 & 10,0 & 10,0 & 10,0 \\
\cline { 2 - 6 } & Poco & 2 & 10,0 & 10,0 & 20,0 \\
\cline { 2 - 6 } & Ni mucho ni poco & 4 & 20,0 & 20,0 & 40,0 \\
\cline { 2 - 6 } & Mucho & 9 & 45,0 & 45,0 & 85,0 \\
\cline { 2 - 6 } & Bastante & 3 & 15,0 & 15,0 & 100,0 \\
\cline { 2 - 6 } & Total & 20 & 100,0 & 100,0 & \\
\hline
\end{tabular}


En relación a la temática educativa trabajada a través del arte, para el $80 \%$ esta experiencia ha supuesto una reflexión sobre la coeducación y la situación de la mujer en la sociedad actual. En su gran mayoría (85\%) se sienten satisfechos con la experiencia educativa realizada, siete de cada diez creen que les ayudará cuando ejerzan la docencia y todos utilizarán en su futuro profesional el arte como recurso didáctico para conseguir objetivos curriculares. Los resultados obtenidos en nuestra intervención educativa están en concordancia con los obtenidos en otras experiencias similares que han utilizado la pedagogía artística en el diseño y creación de recursos didácticos (Mundet et al., 2015; Vidagañ et al., 2016; Orr y Shreeve, 2017; Winner et al., 2014).

\section{DISCUSION FINAL}

La sociedad del Siglo XXI, más que nunca, exige que los estudiantes universitarios adquieran unas competencias personales y profesionales que les permitan enfrentarse a la vida laboral, y también, saber desenvolverse en el mundo con una actitud crítica y una alfabetización socio cultural y educativa, que les posibilite enfrentarse a los cambios, desde el punto de vista de la innovación y la creatividad. De acuerdo con Winner et al. (2014), el bienestar y la felicidad de las personas serán mayores en los países donde al arte le brinde un papel prominente en las escuelas. Por otro lado, las últimas investigaciones, en relación con los efectos sociales del arte, encuentran correlaciones positivas entre la autoestima, la creatividad y la empatía (Mika y Koo, 2020).

De acuerdo con la investigación realizada por Akyıldız y Çelik (2020), los docentes no conocen y no aplican el enfoque creativo en sus asignaturas, no obstante, realizan de forma excepcional juegos y actividades creativas. En este trabajo también se pone de manifiesto que hay factores limitantes de la creatividad como el curriculum, los horarios y los exámenes. Así mismo existen factores facilitadores como la motivación del profesor y la tecnología.

En concordancia con las investigaciones actuales, nuestra propuesta didáctica aúna el desarrollo creativo con las Tecnologías de la Información y la Comunicación (TIC) mediante la técnica de animación Cut out, desarrollando el foco del aprendizaje no sólo en metodología o técnicas sino entendiendo la creatividad como un proceso de autorreflexión individual del alumnado y posibilitando la creación de materiales didácticos que puedan ser utilizados en otros contextos educativos para trabajar la sensibilización y la igualdad de género. Es muy difícil poder dar respuesta a las necesidades con las que nuestros alumnos se encontrarán en su futuro, necesidades que ni siquiera podemos llegar a intuir en una sociedad que evoluciona cada día más rápido, lo que si podemos hacer desde la formación universitaria es desarrollar la competencia creativa de forma transversal. En esta experiencia, la utilización de las TIC en vinculación con la pedagogía del arte se ha convertido en una excelente combinación que ha contribuido a desarrollar la competencia creativa, tan necesaria en los profesionales de la educación.

\section{CONCLUSIONES}

De acuerdo al trabajo presentado y a los resultados obtenidos, se pueden plantear las siguientes conclusiones principales:

1. La utilización de la pedagogía del arte a través de un currículum centrado en propuestas artísticas y creativas contribuye a la motivación del alumnado hacia la reflexión y los contenidos propuestos, esta es una de las principales conclusiones a las que hemos podido llegar en nuestra investigación. Sin embargo, se suele utilizar el arte como propuesta plástica más que como una experiencia reflexiva y aunque esta parte de la experiencia artística posee interesantes beneficios sobre el desarrollo de la motricidad fina y el desarrollo de la percepción estética, si estas actividades son bien planificadas, también contribuirán al desarrollo de la creatividad.

2. Según Maeso-Rubio (2007) vivimos tiempos difíciles dentro del sistema educativo, los altos índices de fracaso escolar obligan a plantear diferentes estrategias basándonos en la educación en valores, haciendo de nuestros contenidos argumentos críticos para que nuestros alumnos tengan una formación completa. Experiencias metodológicas como la presentada en este artículo hacen desarrollar en el futuro profesional de la pedagogía componentes madurativos críticos sin los cuales no podríamos despertar la conciencia social, tan necesaria en el ámbito educativo.

3. Una vez revisada la literatura existente, concluimos que la experiencia de innovación docente utilizando la pedagogía del arte enmarcada en el proyecto IDDEA pretende dar respuesta a una de las problemáticas del curriculum actual, el cual carece de una perspectiva de género que es importante investigar y para la que es preciso proponer pedagogías integradoras, en la línea de los planteamientos de Jiménez-Quenguan y Galeano-Barbosa (2020). 


\section{REFERENCIAS}

Acaso, M. y Megías, C., Art thinking. Transformar la educación a través de las artes, by Paidós Educación, Barcelona, España (2017).

Akyıldız, S.T. y Çelik, V. Thinking outside the box: Turkish EFL teachers' perceptions of creativity, doi: 10.1016/j.tsc.2020.100649, Thinking Skills and Creativity, 36 (2020).

Berrocal, M., Caja, J y González, J. M, Educar la mirada, la mano y el pensamiento; in La educación visual y plástica hoy. Educar la mirada, la mano y el pensamiento, by Editorial Graó, pp 59-79, Barcelona, España (2010).

Cropley, D.H., Patston, T., Marrone, R.L. y Kaufman, J.C., Essential, unexceptional and universal: teacher implicit beliefs of creativity, doi: 10.1016/j.tsc.2019.100604, Thinking Skills and Creativity, 34, (2019).

Delgado Benito, V., Ruiz Franco, M., Ausín Villaverde, V y Abella García, V, Píldoras formativas audiovisuales, un recurso didáctico multimedia a través de la metodología educativa "Learning by Doing"; in Diferentes perspectivas de la educación del siglo XXI, by Asire Educación, pp. 17-28, Burgos, España (2015).

Hennessey, B.A., Creativity, doi: 10.1146/annurev.psych.093008.100416, Annual Review of Psychology, 61 (10), 569-598 (2010).

Holdhus, K., When students teach creativities: Exploring student reports on creative teaching, doi: 10.1177/1077800418801377, Qualitative Inquiry, 25(7), 690-699 (2019).

Jeffrey, B. y Craft, A., Teaching creatively and teaching for creativity: distinctions and relationships, doi: 10.1080/0305569032000159750, Educational Studies, 30(1), 77-87 (2004).

Jiménez-Quenguan, M., y Galeano-Barbosa, D. J., La necesidad de educar en perspectiva de género, doi: 10.15517/revedu.v44i1.38529, Revista Educación, 44(1), 490-508 (2020).

Livingston, L., Teaching creativity in higher education, doi: 10.1080/10632910903455884, Arts Education Policy Review, 2, 59-62 (2010).

Loveless, A., Making a difference? an evaluation of professional knowledge and pedagogy in art and ICT, doi: 10.1111/1468-5949.00350, International Journal of Art \& Design Education, 22(2), 145-154 (2003).

Maeso-Rubio, F, Aprender a enseñar las artes visuales desde una perspectiva crítica y construccionista, in El arte de enseñar el arte. Metodología innovadora en bellas artes, by Diferencia, pp. 129-152, Sevilla, España (2008).

Maeso-Rubio, F., La TV y la educación en valores, doi: 10.3916/c31-2008-03-028, Comunicar: Revista Científica de Comunicación y Educación, 16(31), 417-421 (2008).

Mayo, S., Implications for art education in the third millennium: art technology integration, doi: 10.1080/00043125.2007.11651644, Art Education, 60 (3), 45-51 (2007).

Menéndez-Varela, J.L. y Grigori-Giralt, E., La construcción de la identidad profesional de estudiantes universitarios de arte a través de proyectos de aprendizaje-servicio, doi: 10.5209/ARIS.55249, Arte, Individuo y Sociedad, 29(3), 417-443 (2017).

Mika, J. y Koo, S., The Influence of Cultural and Art Activities on Social Interaction of Teenagers - A Case of a Youth Art School in Busan Jin-gu, doi: 10.3389/fpsyg.2019.00537, The Korean Journal of Arts Studies, 27, $311-342$ (2020)

Mora, J. M y Osses, S., Educación artística para la formación integral. Complementariedad entre cultura visual e identidad juvenil, doi: 10.4067/S0718-07052012000200020, Estudios Pedagógicos. 38(2), 321-335 (2012)

Moreno-González, A. M., La mediación artística: un modelo de educación artística para la intervención social a través del arte. Revista Iberoamericana de Educación, ISSN: 1022-6508, 52(2), 1-9 (2010).

Moreno, J.R., Vera, M.I. y López, I., Development of creative and educational thinking in arts training teachers: QR codes, doi: 10.5281/zenodo.34305, Sylman Journal, 158(12), 185-200 (2014).

Mundet, A., Beltrán, A. Ma . y Moreno, A., Arte como herramienta social y educativa, doi: 10.5209/rev_RCED.2015.v26.n2.43060, Revista Complutense de Educación, 26(2), 315-329 (2015).

Orr, S. y Shreeve, A., Art and design pedagogy in higher education: Knowledge, values and ambiguity in the creative curriculum, 140-155, Routledge, New York, EEUU (2017).

París, G., 5×5×5=Creativity: Art as a Transformative Practice, doi: 10.1111/jade.12229, International Journal of Art and Design Education. 39 (1), 69-84 (2020).

Pavlou, V., Art technology integration: digital storytellying as a transformative pedagogy in primary education. doi: 10.1111/jade.12254, International Journal of Art and Design Education. 39(1), 195-210 (2020).

Robinson, K y Aronica, L, 1르. Ed., You, Your child, and school: navigate your way to the best education, Penguin. LCC US, Londres, Inglaterra (2019).

Orr, S. y Shreeve, A., Art and design pedagogy in higher education: Knowledge, values and ambiguity in the creative curriculum, 140-155, Routledge, New York, EEUU (2017). 
Suárez, N., Delgado, K. E., Pérez, I. C. y Barba, M. N., Desarrollo de la Creatividad y el Talento desde las Primeras Edades. Componentes Curriculares de un Programa de Maestría en Educación, doi: 10.4067/S071850062019000600115, Formación Universitaria, 12(6), 115-126 (2019).

Touriñán, J. M., Educación artística: sustantivamente "educación" y adjetivamente "artística", doi: 10.5944/educxx1.16453, Educación XXI, 19(2), 45-76 (2016).

Ucus, S. y Acar, I.H., Exploring the perceptions of student teachers about 'creative school' in Early Childhood Education, doi: 10.1080/03004430.2017.1307838, Early Child Development and Care, 189(2),191-206 (2019).

Vidagañ, M., Soria, V. y Lozano, N., Arte y educación: espacios comunes. Reflexiones en torno a la práctica artística Mapa dels desitjos. Educación artística, doi: 10.7203/eari.7.8071, Revista de Investigación, 7, 135-145 (2016).

Whitaker, A., Art thinking: how to carve out creative space in a world of schedules, budgets and bosses, 120-156, Harper Collins Publishers, New York, EEUU (2016).

Winner, E., Goldsteis, T. y Vicent-Lancris, S., ¿En arte por el arte? La influencia de la educación artística, Instituto Politécnico Nacional, México, América del Norte (2014). 
